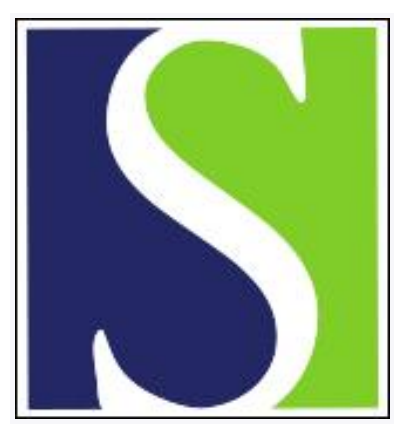

Scand J Work Environ Health 1992;18(2):124-127

https://doi.org/10.5271/sjweh.1601

Issue date: 01 Apr 1992

Sympathetic nerve activity in the skin in relation to vibration-induced white finger.

by Ishida G, Nasu Y, Nakashima K, Sato T, Takahashi K

Affiliation: Division of Neurology, Faculty of Medicine, Tottori University, Yonago, Japan.

This article in PubMed: www.ncbi.nlm.nih.gov/pubmed/1604273 


\title{
Sympathetic nerve activity in the skin in relation to vibration-induced white finger
}

\author{
by Gen Ishida, MD, ${ }^{1}$ Yoshiro Nasu, MD, ${ }^{2}$ Kenji Nakashima, MD, ${ }^{1}$ Takeo Sato, MD, ${ }^{3}$ \\ Kazuro Takahashi, MD'
}

\begin{abstract}
ISHIDA G, NASU Y, NAKASHIMA K, SATO T, TAKAHASHI K. Sympathetic nerve activity in the skin in relation to vibration-induced white finger. Scand $J$ Work Environ Health 1992;18:124-7. With a microneurographic method, sympathetic nerve activity in the skin was recorded from median nerve fascicles of six patients with vibration-induced white finger and four age- and gender-matched normal subjects. At an ambient temperature of $15^{\circ} \mathrm{C}$ the sympathetic nerve activity was greater in the patients than in the normal subjects. The burst activity was increased in all of the subjects during the water immersion test of the contralateral hand, but neither the increase in the mean burst number nor the mean area under the neurogram showed statistically significant group differences. Thus, although vasoconstrictive tone is greater in patients with vibration-induced white finger than in healthy subjects, no support could be found for the hypothesis that increased vascular tone is of prime importance for attacks of white finger in vibration-exposed subjects.
\end{abstract}

Key terms: microneurography, Raynaud's phenomenon, water immersion test.

Long-term use of hand-held vibrating tools has been recognized as a cause of Raynaud's phenomenon. Many pathogenic theories have been proposed for vibration-induced white finger (VWF). In 1862, Raynaud (1) suggested that the dysfunction in question is due to an increased sympathetic outflow to the vascular bed, while Lewis (2) developed the "local fault" theory.

Recently, humoral and local factors have been emphasized. Some reports have indicated that subjects with VWF have higher viscosity of the circulating blood than subjects without VWF $(3,4)$. Lafferty et al (5) hypothesized peripheral vasospasm as a result of an altered interaction between local sympathetic nervous hyperreactivity to histamine release and sympathetic neural discharges. Methodologically, direct recording of sympathetic nerve activity in the skin with a microneurographic technique was introduced in 1972 (6). This method provides direct access to sympathetic nerve activity in alert subjects.

The present study was designed to compare the level of sympathetic nerve activity in the skin of patients with VWF with that of a group of referents. For this purpose, the sympathetic nerve activity of both patients with VWF and healthy subjects who had not yet been

\footnotetext{
1 Division of Neurology, Institute of Neurological Sciences, Faculty of Medicine, Tottori University, 86 Nishimachi, Yonago 683, Japan.

2 Department of Orthopedic Surgery, San-in Rousai Hospital, 1-8-1 Kaikeshinden, Yonago 683, Japan.

3 Department of Neurology, San-in Ruosai Hospital, 1-8-1 Kaikeshinden, Yonago 683, Japan.
}

Reprint requests to: Dr G Ishida, Division of Neurology, Institute of Neurological Sciences, Faculty of Medicine, Tottori University, 86 Nishimachi, Yonago 683, Japan. exposed to vibratory tools was measured before, during, and after cold water immersion.

\section{Subjects and methods}

Six of the subjects were patients with VWF, aged 52-65 (mean 60.2, SD 4.9) years, and four were ageand gender-matched referents, aged 48-68 (mean 55.5, SD 9.6) years. All of the subjects were men. The length of exposure to vibrating tools for the patients with VWF ranged from 4 to 32 (mean 13.4) years. The patients with VWF were classified according to the revised Taylor-Pelmear's scale (7), which indicated that three were in stage 2 and the rest were in stage 3.

Each subject gave an informed consent to this examination. The following procedures were within the standards of the Committee on Human Experimentation in accordance with the Helsinki Declaration of 1975. The experiment was performed in an air-conditioned, dark and silent room, in which the ambient temperature was kept at $15^{\circ} \mathrm{C}$ so that the sudomotor impulses of sympathetic nerve activity would be suppressed. The subjects lay comfortably in a supine and alert state.

Detailed descriptions of the method used for the nerve potential recording, the recording system, and the essential procedures for finding and identifying sympathetic nerve activity in the skin have been given previously (8). Briefly described, an insulated tungsten microelectrode with a bare tip diameter of $1 \mu \mathrm{m}$ was inserted manually through the skin into the median nerve around the elbow. The characteristic pattern of the multiunits of the sympathetic nerve activity was then located in both original and integrated neuro- 
grams on a thermal dot graph after proper voltage amplification through a high-impedance input preamplifier (WPI Co, New Haven, United States, DAM-6A) and a potential integrator, and simultaneously by monitoring burst tones through a loud speaker. Each potential crest identified as a burst of true sympathetic nerve activity over the noise was marked manually and systematically on the graph of a thermal dot recorder (NEC SAN-EI Co, Tokyo, Japan, 8M36), on which both the original and the integrated neurograms were simultaneously recorded. The time constant of the integrating circuit was $0.1 \mathrm{~s}$. After the sympathetic nerve activity was encountered, spontaneous firing was observed for $5 \mathrm{~min}$. We analyzed the record obtained of the sympathetic nerve activity quantitatively by counting the number of burst crests in the integrated neurogram for each minute. The burst rate was defined as the number of burst crests during $1 \mathrm{~min}$. The contralateral hand was then immersed in cold water $\left(4^{\circ} \mathrm{C}\right)$ for $1 \mathrm{~min}$, and the changes in the burst rate of the sympathetic nerve activity were recorded.

For the integrated neurogram, the area of the respective crests, rather than their number, was considered to reflect changes in the potential amplitude and/or the duration of the bursts. The area under the neurogram (NA) per minute was used as another measure. The areas surrounded for $1 \mathrm{~min}$ by the integrated neurographic line and a base line were measured with an area calculator (Ushikata Co, Tokyo, Japan, X-plan 360). The area was given in arbitrary units (NA unit $/ \mathrm{min}$ ), and the areas during and after immersion were expressed as the percentage of the preimmersion area.

The nerve-potential recording procedure may have caused minor discomfort during the search for the nerve fasciculus. Once a suitable electrode position was found, no discomfort was experienced. The complete examination usually took $2-3 \mathrm{~h}$. Satisfactory activity recordings could be obtained without exception from all of the subjects. Some of the subjects occasionally experienced transient paresthesia for a few days after the recording, but no clinical signs of permanent nerve damage were observed.

The statistical evaluation of the results was carried out with the Wilcoxon rank-sum test for comparisons between normal referents and patients with VWF and also with the paired t-test for comparisons between the various periods.

\section{Results}

Representative examples of the original records of the sympathetic nerve activity and the integrated neurograms of a referent and a patient with VWF are shown in figure 1. The integrated neurograms show that the basic activity bursts were greater in the patient than in the healthy subject, both with and without cold provocation. Furthermore, an increased number of burst crests can be seen during the cold water immersion of the contralateral hand for both the referent and the patient. This finding was common for all of the subjects and may indicate an increase in sympathetic outflow induced by cold water immersion, even though Raynaud's phenomenon was not elicited.

Figure 2 illustrates all of the results in which the measure of the activity burst rate was used. Before the immersion, the average activity burst rate was 24.5 (SD 7.7) burst/min for the referents and 34.8 (SD 6.4) burst/min for the patients with VWF. During the immersion, the average value was 34.0 (SD 5.5) burst $/ \mathrm{min}$ for the referents and 44.0 (SD 5.2) burst/min for the patients with VWF. In both the preimmersion and immersion periods, greater burst rates of sympathetic nerve activity were found for the patients than for the referents $(\mathrm{P}<0.05)$. After immersion, the burst rate of the referents was 27.0 (SD 8.6) burst $/ \mathrm{min}$, while that of the patients with VWF was 36.5 (SD 8.1) burst/min. The group differences were not statistically significant.

For the referents, there were statistically significant differences in the burst rates between the preimmersion and immersion periods $(P<0.01)$ and between the
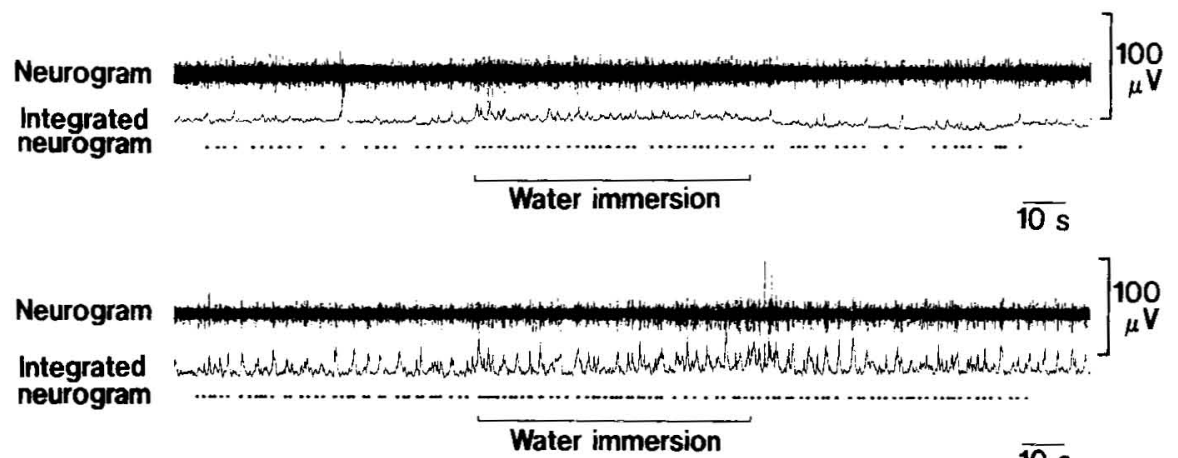

Water immersion

$\widetilde{10 s}$

Figure 1. Representative thermal dot-writing records from a neurogram of sympathetic nerve activity in the skin and an integrated neurogram before, during, and after water immersion (upper traces $=$ a healthy referent, lower traces $=$ a patient with vibration. induced white finger). The rows of dots under the two sets of neurograms correspond in time to the respective burst crests identified as sympathetic nerve activity. 


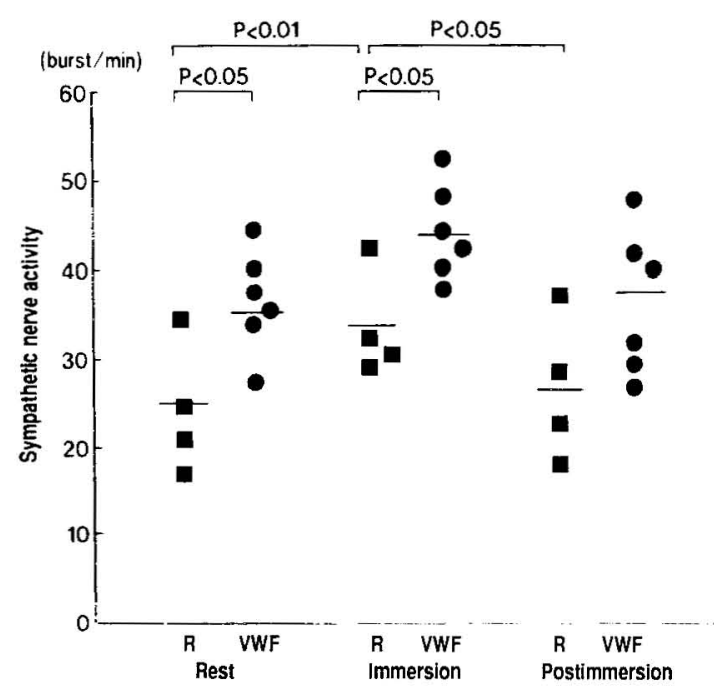

Figure 2. Burst rates of sympathetic nerve activity in the skin before, during, and after water immersion. $(R=$ referents, $\mathrm{VWF}=$ patients with vibration-induced white finger)

immersion and postimmersion periods $(\mathrm{P}<0.05)$, but no difference was found between the periods of preand postimmersion. For the patients with VWF, there were no statistically significant differences between the three periods. The increment in the sympathetic nerve activity induced by cold water immersion was 38.8 (range 20.0-82.4) $\%$ of the preimmersion level for the referents and 26.4 (range $4.5-77.8$ ) $\%$ for the patients. The group difference was not statistically significant.

During provocation by cold water immersion, not only the number of neural discharges, but also their respective amplitude and duration seemed to be increased. Figure 3 illustrates the results in which the area under the integrated neurogram was used as another indication of the change in the bursts of sympathetic nerve activity. The mean value during immersion was 162 (range $141-180) \%$ of the preimmersion level for the referents and $148(131-165) \%$ for the VWF patients. At the end of the recording period, after the immersion (maximum $2 \mathrm{~min}$ ), the corresponding values were 115 (range 94-150)\% for the referents and 108 $(100-121) \%$ for the patients. These group differences during and also after the immersion were not statistically significant.

\section{Discussion}

The sympathetic nerve activity in the skin consists of vasoconstrictor and sudomotor impulses (6). The two types of fibers are intermingled, and it is difficult to record pure vasoconstrictor impulses isolated from sudomotor impulses. Changing the environmental temperature makes it possible to obtain a selective activa-

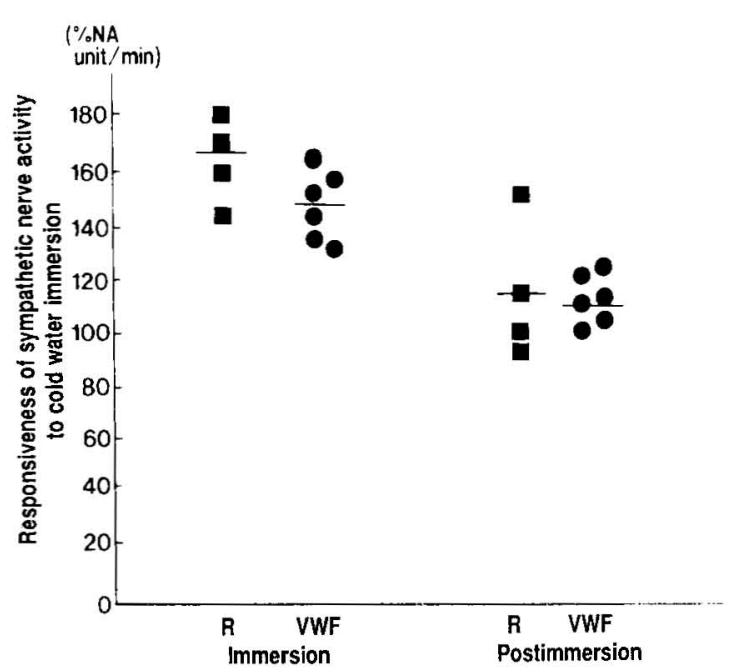

Figure 3. Percentage of change (from the preimmersion level) in the sympathetic nerve activity in the skin during and after water immersion, expressed as the area under the integrated neurogram (NA unit) per minute. ( $R=$ referents, VWF = patients with vibration-induced white finger)

tion of vasoconstrictor impulses only (9). In other words, with a lower ambient temperature, the vasoconstrictor impulses become more dominant because the sudomotor impulses are suppressed. Our experimental room temperature was maintained at $15^{\circ} \mathrm{C}$, and the subjects were adequately adapted to the ambient temperature before the nerve potential recordings. Therefore, it may be safely said that the recorded activity of the sympathetic nerve represented only vasoconstrictor impulses in this examination.

In this experiment, the sympathetic tone of the patients was shown to be higher than that of the referents before and during the cold provocation. Since there was no evidence of a greater increase in neurogram area for the patients than for the referents, our results failed, however, to support the hypothesis that increased activity of the sympathetic nervous system is of primary importance to the triggering of Raynaud's phenomenon.

\section{Acknowledgments}

We thank Ms S Ito for her valuable help in the preparation of the paper.

\section{References}

1. Raynaud M. De láasphyxie locale et de la gangrène symmétryque des extrémités. Paris: Rignoux, 1862.

2. Lewis T. Experiments relating to the peripheral mechanism involved in spasmodic arrest of the circulation in the fingers, a variety of Raynaud's disease. Heart 1929; 15:7-102.

3. Ikehata K, Kawauchi S, Kohno F. Increased platelet 
function and von Willebrand factor in vibration syndrome. Tokushima J Exp Med 1980;27:23-8.

4. Okada A, Yamashita T, Nagano C, Ikeda T, Yachi A, Shibata S. Studies on the diagnosis and pathogenesis of Raynaud's phenomenon of occupational origin. $\mathrm{Br} \mathrm{J}$ Ind Med 1971;28:353-7.

5. Lafferty K, De Trafford JC, Roberts VC, Cotton LT On the nature of Raynaud's phenomenon: the role of histamine. Lancet 1983:2;313-5.

6. Hagbarth KE, Hallin RG, Hongel A, Torebjörk HE, Wallin BG. General characteristics of sympathetic activity in human skin nerves. Acta Physiol Scand 1972; 84:164-76.

7. Gemne G, Pyykkö I, Taylor W, Pelmear PL. The Stock- holm Workshop scale for the classification of coldinduced Raynaud's phenomenon in the hand-arm vibration syndrome (revision of the Taylor-Pelmear scale). Scand J Work Environ Health 1987;13:275-8.

8. Ishida G, Nakashima K, Takahashi K. Skin nerve sympathetic activity reflex latency in Parkinson's disease. Acta Neurol Scand 1990;81:121-4.

9. Bini G, Hagbarth KE, Hynninen P, Wallin BG. Thermoregulatory and rhythm-generating mechanisms governing the sudomotor and vasoconstrictor outflow in human cutaneous nerves. J Physiol 1980;306:537-52.

Received for publication: 28 December 1991 